\title{
Diagonal approximation and the cohomology ring of the fundamental groups of surfaces
}

\author{
Daciberg Lima Gonçalves • Sérgio Tadao Martins
}

Received: 15 May 2014 / Revised: 7 September 2014 / Accepted: 30 October 2014 /

Published online: 6 January 2015

(C) Springer International Publishing AG 2015

\begin{abstract}
We construct finite free resolutions of $\mathbb{Z}$ over $\mathbb{Z} \pi$, where $\pi$ is the fundamental group of a surface distinct from $S^{2}$ and $\mathbb{R} P^{2}$, and define diagonal approximations for these resolutions. We then proceed to give some applications using the knowledge of those maps.
\end{abstract}

Keywords Surface - Group cohomology · Diagonal approximation ·

Local coefficients · Bundles

Mathematics Subject Classification $\quad 20 \mathrm{~J} 06 \cdot 57 \mathrm{M} 20 \cdot 55 \mathrm{R} 15$

\section{Introduction}

The closed surfaces other than $S^{2}$ and $\mathbb{R} P^{2}$ are $K(\pi, 1)$ spaces, which means that their cohomology rings coincide with the cohomology rings of their fundamental groups. There are several situations where one may be interested in the cohomology groups of surfaces, notoriously with non-trivial local coefficients. See, for instance,

The paper was originally submitted on 17 October 2013 to the Central European Journal of Mathematics and withdrawn on 2 April 2014 due to imposing of publishing fees.

Sérgio Tadao Martins has received financial support from FAPESP, processes 2013/07510-4 and 2013/21394-7.

D. L. Gonçalves $(\bowtie) \cdot$ S. T. Martins

Department of Mathematics-IME, University of São Paulo, Rua do Matão 1010,

São Paulo 05508-090, Brazil

e-mail: dlgoncal@ime.usp.br

S. T. Martins

e-mail: sergiotm@ime.usp.br 
[7, Part I, Chapter 5], [4] and in general obstruction theory. Given that interest, in this paper we construct finite free resolutions for fundamental groups of $K(\pi, 1)$ surfaces and partial diagonal approximations for these resolutions, which allow us to compute the cohomology rings $H^{*}(\pi, M)$ for any coefficient $M$ in an efficient way.

Let us briefly recall how to define and compute (at least in theory) not only the cohomology groups $H^{*}(\pi, M)$ for a given group $\pi$ with coefficients in the $\mathbb{Z} \pi$-module $M$, but also how to determine the multiplicative structure given by the cup product. More details about the definitions can be found in [2].

If $M$ is a (left) $\mathbb{Z} \pi$-module, the cohomology group $H^{n}(\pi, M)$ is defined by $H^{n}(\pi, M)=\operatorname{Ext}_{\mathbb{Z} \pi}^{n}(\mathbb{Z}, M)$ for $n \geq 0$, where $\mathbb{Z}$ is the trivial $\mathbb{Z} \pi$-module. Hence one way to compute the groups $H^{n}(\pi, M)$ is this: first, we find a projective resolution $P$ over the ring $\mathbb{Z} \pi$ of the trivial $\mathbb{Z} \pi$-module $\mathbb{Z}$. Then we apply the functor $\operatorname{Hom}_{\mathbb{Z} \pi}(\cdot, M)$ to the chain complex $P$ and the cohomology groups $H^{n}(\pi, M)$ are the cohomology groups of the chain complex $\operatorname{Hom}_{\mathbb{Z} \pi}(P, M)$. Also, if

$$
\cdots \rightarrow P_{n} \stackrel{d_{n}}{\rightarrow} P_{n-1} \rightarrow \cdots \rightarrow P_{0} \stackrel{\varepsilon}{\rightarrow} \mathbb{Z} \rightarrow 0
$$

is a projective resolution of $\mathbb{Z}$, then

$$
\cdots \rightarrow(P \otimes P)_{n} \stackrel{\partial_{n}}{\longrightarrow}(P \otimes P)_{n-1} \rightarrow \cdots \rightarrow(P \otimes P)_{0} \stackrel{\varepsilon \otimes \varepsilon}{\longrightarrow} \mathbb{Z} \otimes \mathbb{Z} \cong \mathbb{Z} \rightarrow 0
$$

is also a projective resolution, and there is a map of chain complexes $\Delta: P \rightarrow(P \otimes P)$ such that

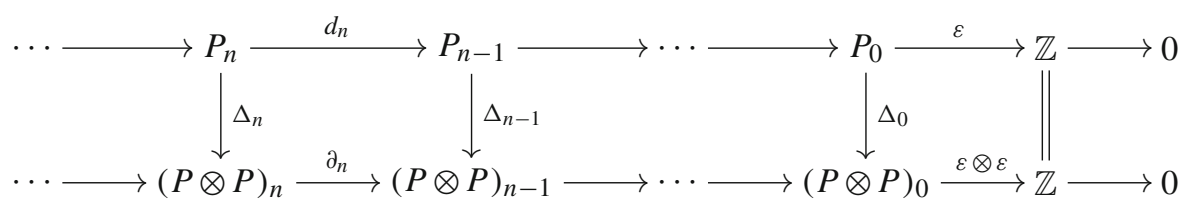

is commutative. The map $\Delta$ is called a diagonal approximation for the resolution $P$ and is used to define the cup product

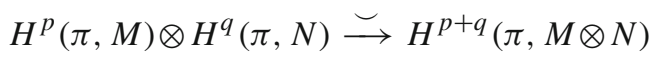

in the following way: let $u \in \operatorname{Hom}_{\mathbb{Z} \pi}\left(P_{p}, M\right), v \in \operatorname{Hom}_{\mathbb{Z} \pi}\left(P_{q}, N\right)$, and let $\alpha \in$ $H^{p}(G, M)$ and $\beta \in H^{q}(\pi, N)$ be the classes of homomorphisms $u$ and $v$, respectively. The cup product of $\alpha$ and $\beta$ is defined by

$$
(\alpha \smile \beta)=[(u \otimes v) \circ \Delta] \in H^{p+q}(\pi, M \otimes N),
$$

where $[\cdot]$ denotes the cohomology class. Given that definition, we can be a little more specific: the product $(\alpha \smile \beta)$ in $(1)$ is the cohomology class of the map $(u \otimes v) \circ \Delta_{p q}$, 
where $\Delta_{p q}: P_{p+q} \rightarrow P_{p} \otimes P_{q}$ is the composition of $\Delta_{p+q}: P_{p+q} \rightarrow(P \otimes P)_{p+q}$ with the projection $\pi_{p q}:(P \otimes P)_{p+q} \rightarrow\left(P_{p} \otimes P_{q}\right)$.

Thus, the computation of cohomology groups $H^{*}(\pi, M)$ together with the multiplicative structure given by the cup product can be accomplished if we manage to find a free resolution $P$ and a diagonal approximation $\Delta$ for the resolution $P$. This is not an easy task in general. In [6] we find the following two propositions, which can help us to determine $\Delta$.

Proposition 1.1 For a group $\pi$, let

$$
\cdots \rightarrow C_{n} \rightarrow \cdots \rightarrow C_{1} \rightarrow C_{0} \stackrel{\varepsilon}{\rightarrow} \mathbb{Z} \rightarrow 0
$$

be a free resolution of $\mathbb{Z}$ over $\mathbb{Z} \pi$. If $s$ is a contracting homotopy for the resolution $C$, then a contracting homotopy $\widetilde{s}$ for the free resolution $C \otimes C$ of $\mathbb{Z}$ over $\mathbb{Z} \pi$ is given by

$$
\begin{aligned}
\widetilde{s}_{-1}: & \mathbb{Z} \rightarrow C_{0} \otimes C_{0} \\
& \widetilde{s}_{-1}(1)=s_{-1}(1) \otimes s_{-1}(1), \\
\widetilde{s}_{n}: & (C \otimes C)_{n} \rightarrow(C \otimes C)_{n+1} \\
& \widetilde{s}_{n}\left(u_{i} \otimes v_{n-i}\right)=s_{i}\left(u_{i}\right) \otimes v_{n-i}+s_{-1} \varepsilon\left(u_{i}\right) \otimes s_{n-i}\left(v_{n-i}\right) \quad \text { if } n \geq 0,
\end{aligned}
$$

where $s_{-1} \varepsilon: C_{0} \rightarrow C_{0}$ is extended to $s_{-1} \varepsilon=\left\{\left(s_{-1} \varepsilon\right)_{n}: C_{n} \rightarrow C_{n}\right\}$ in such a way that $\left(s_{-1} \varepsilon\right)_{n}=0$ for $n \geq 1$.

Proposition 1.2 For a group $\pi$, let

$$
\cdots \rightarrow C_{n} \stackrel{d_{n}}{\rightarrow} \cdots \rightarrow C_{1} \stackrel{d_{1}}{\rightarrow} C_{0} \stackrel{\varepsilon}{\rightarrow} \mathbb{Z} \rightarrow 0
$$

be a free resolution of $\mathbb{Z}$ over $\mathbb{Z} \pi$. Let $B_{n}$ be a $\mathbb{Z} \pi$-basis for $C_{n}$ for each $n \geq 0$ such that $\varepsilon(b)=1$ for all $b \in B_{0}$, and let $s$ be a contracting homotopy for this resolution $C$. If $\widetilde{s}$ is the contracting homotopy for the resolution $C \otimes C$ given by Proposition 1.1, then a diagonal approximation $\Delta: C \rightarrow C \otimes C$ can be defined in the following way: for each $n \geq 0$, the map $\Delta_{n}: C_{n} \rightarrow(C \otimes C)_{n}$ is given on each element $\rho \in B_{n} \subset C_{n}$ by

$$
\Delta_{0}=s_{-1} \varepsilon \otimes s_{-1} \varepsilon, \quad \Delta_{n}(\rho)=\widetilde{s}_{n-1} \Delta_{n-1} d_{n}(\rho) \quad \text { if } n \geq 1 .
$$

Essentially, the above propositions tell us that if we have a finitely generated free resolution $P$ of $\mathbb{Z}$ over $\mathbb{Z} \pi$, then a diagonal approximation $\Delta: P \rightarrow(P \otimes P)$ can be calculated if we have a contracting homotopy for the resolution $P$. This is the approach we will use to determine partial diagonal approximations for the resolutions of fundamental groups of $K(\pi, 1)$ surfaces. The word "partial" has the following meaning: since we are dealing with $K(\pi, 1)$ surfaces, the finitely generated free resolution $P$ is given by the augmented cellular chain complex of the universal cover $\mathbb{R}^{2}$ of the surface as a free $\mathbb{Z} \pi$-complex. As the comohology groups $H^{n}(\pi, M)$ are then trivial for $n \geq 3$, the observation after (1) tells that we only need the maps $\Delta_{0}: P_{0} \rightarrow\left(P_{0} \otimes P_{0}\right)$, 
$\Delta_{01}: P_{1} \rightarrow\left(P_{0} \otimes P_{1}\right), \Delta_{02}: P_{2} \rightarrow\left(P_{0} \otimes P_{2}\right)$ and $\Delta_{11}: P_{2} \rightarrow\left(P_{1} \otimes P_{1}\right)$ to compute the meaningful products $H^{p}(\pi, M) \otimes H^{q}(\pi, N) \rightrightarrows H^{p+q}(\pi, M \otimes N)$.

One last remark before we move to the actual computations in the next section: given any group $\pi$ and any projective resolution $P$ of $\mathbb{Z}$ over $\mathbb{Z} \pi$, a contracting homotopy $s$ for the resolution $P$ can be constructed "piece by piece", as the next lemma shows.

Lemma 1.3 Let $\pi$ be a group and

$$
\cdots \rightarrow P_{n} \stackrel{d_{n}}{\rightarrow} P_{n-1} \rightarrow \cdots \rightarrow P_{0} \stackrel{\varepsilon}{\rightarrow} \mathbb{Z} \rightarrow 0
$$

a projective resolution of $\mathbb{Z}$ over $\mathbb{Z} \pi$. Suppose that, for a given $n \geq 0$, there are maps of abelian groups $s_{k}: P_{k} \rightarrow P_{k+1}$ for $-1 \leq k \leq n$ such that $d_{k+1} s_{k}+s_{k-1} d_{k}=\mathrm{id}_{P_{k}}$ for all $-1 \leq k \leq n$ (we let $P_{-1}=\mathbb{Z}$ ). Then there is a map of abelian groups $s_{n+1}: P_{n+1} \rightarrow P_{n+2}$ such that $d_{n+2} s_{n+1}+s_{n} d_{n+1}=\mathrm{id}_{P_{n+1}}$.

Proof If $P_{n+1}=0$, then $s_{n+1}=s_{n}=0$ and there is nothing to prove. If $P_{n+1} \neq 0$, let $w$ be an element of a basis of $P_{n+1}$, viewed as a free abelian group. It suffices to show that we can find $y \in P_{n+2}$ such that $d_{n+2}(y)+s_{n} d_{n+1}(w)=w$, since we can then define $s_{n+1}(w)=y$. Then all we have to do is to prove that $w-s_{n} d_{n+1}(w) \in$ $\operatorname{im}\left(d_{n+2}\right)=\operatorname{ker}\left(d_{n+1}\right)$. But

$$
\begin{aligned}
d_{n+1}\left(w-s_{n} d_{n+1}(w)\right) & =d_{n+1}(w)-d_{n+1} s_{n} d_{n+1}(w) \\
& =d_{n+1}(w)-\left(d_{n+1}(w)-s_{n-1} d_{n} d_{n+1}(w)\right) \\
& =d_{n+1}(w)-d_{n+1}(w)=0 .
\end{aligned}
$$

In Sect. 2 we use the results above to determine diagonal approximations for the fundamental groups of $K(\pi, 1)$ surfaces, and in Sect. 3 we make some comments on applications.

\section{Free resolutions and diagonal approximations}

Let $M_{n}$ be the orientable surface of genus $n \geq 1$, with fundamental group $G$ given by the presentation

$$
G=\pi_{1}\left(M_{n}\right)=\left\langle a_{1}, b_{1}, a_{2}, b_{2}, \ldots, a_{n}, b_{n} \mid\left[a_{1}, b_{1}\right]\left[a_{2}, b_{2}\right] \cdots\left[a_{n}, b_{n}\right]\right\rangle .
$$

We also let $p_{i}=\left[a_{i}, b_{i}\right]=a_{i} b_{i} a_{i}^{-1} b_{i}^{-1}$ and $p=p_{1} p_{2} \cdots p_{n}$.

Proposition 2.1 (free resolution for the orientable case) A free resolution of $\mathbb{Z}$ over $\mathbb{Z} G$ is given by

$$
0 \rightarrow P_{2} \stackrel{d_{2}}{\rightarrow} P_{1} \stackrel{d_{1}}{\rightarrow} P_{0} \stackrel{\epsilon}{\rightarrow} \mathbb{Z} \rightarrow 0
$$

where

$$
P_{0}=\langle x\rangle \cong \mathbb{Z} G, \quad P_{1}=\left\langle y_{1}, \ldots, y_{n}, z_{1}, \ldots, z_{n}\right\rangle \cong \mathbb{Z} G^{2 n}, \quad P_{2}=\langle w\rangle \cong \mathbb{Z} G,
$$


and the maps $\varepsilon, d_{1}$ and $d_{2}$ are defined by

$$
\begin{gathered}
\varepsilon(x)=1, \quad d_{1}\left(y_{i}\right)=\left(a_{i}-1\right) x, \quad d_{1}\left(z_{i}\right)=\left(b_{i}-1\right) x, \\
d_{2}(w)=\sum_{i=1}^{n}\left(\frac{\partial p}{\partial a_{i}} y_{i}+\frac{\partial p}{\partial b_{i}} z_{i}\right)
\end{gathered}
$$

where the partial derivatives are the Fox derivatives.

Proof Since the surface $M_{n}$ is a $K(G, 1)$ space, its universal cover $\mathbb{R}^{2}$ is a free and contractible $G$-complex. Hence its augmented cellular chain complex, which is proved to be exactly (2) in [3], is a free resolution of $\mathbb{Z}$ over $\mathbb{Z} G$.

We now proceed to construct a diagonal approximation $\Delta: P \rightarrow(P \otimes P)$ for the resolution $P$ given by the above proposition.

Theorem 2.2 (diagonal approximation for the orientable case) Let $P$ be the free resolution of $\mathbb{Z}$ over $\mathbb{Z} G$ given by Proposition 2.1. A diagonal approximation $\Delta: P \rightarrow$ $(P \otimes P)$ is partially given by

$$
\begin{aligned}
& \Delta_{0}: P_{0} \rightarrow(P \otimes P)_{0} \quad \Delta_{0}(x)=x \otimes x, \\
& \Delta_{1}: P_{1} \rightarrow(P \otimes P)_{1} \quad \Delta_{1}\left(y_{i}\right)=y_{i} \otimes a_{i} x+x \otimes y_{i}, \quad \Delta_{1}\left(z_{i}\right)=z_{i} \otimes b_{i} x+x \otimes z_{i}, \\
& \Delta_{02}: P_{2} \rightarrow\left(P_{0} \otimes P_{2}\right) \quad \Delta_{02}(w)=x \otimes w, \\
& \Delta_{11}: P_{2} \rightarrow\left(P_{1} \otimes P_{1}\right) \\
& \Delta_{11}(w)=\sum_{i=1}^{n}\left[\sum_{j=1}^{i-1}\left(p_{1} \cdots p_{j-1}\right)\left(1-a_{j} b_{j} a_{j}^{-1}\right) y_{j} \otimes\left(p_{1} \cdots p_{i-1}\right) y_{i}\right. \\
& \left.+\sum_{j=1}^{i-1}\left(p_{1} \cdots p_{j-1}\right) a_{j}\left(1-b_{j} a_{j}^{-1} b_{j}^{-1}\right) z_{j} \otimes\left(p_{1} \cdots p_{i-1}\right) y_{i}\right] \\
& -\sum_{i=1}^{n-1}\left[\sum _ { j = 1 } ^ { i - 1 } \left(\left(p_{1} \cdots p_{j-1}\right)\left(1-a_{j} b_{j} a_{j}^{-1}\right) y_{j} \otimes\left(p_{1} \cdots p_{i-1}\right) a_{i} b_{i} a_{i}^{-1} y_{i}\right.\right. \\
& \left.+\left(p_{1} \cdots p_{j-1}\right) a_{j}\left(1-b_{j} a_{j}^{-1} b_{j}^{-1}\right) z_{j} \otimes\left(p_{1} \cdots p_{i-1}\right) a_{i} b_{i} a_{i}^{-1} y_{i}\right) \\
& +\left(p_{1} \cdots p_{i-1}\right)\left(1-a_{i} b_{i} a_{i}^{-1}\right) y_{i} \otimes\left(p_{1} \cdots p_{i-1}\right) a_{i} b_{i} a_{i}^{-1} y_{i} \\
& \left.+\left(p_{1} \cdots p_{i-1}\right) a_{i} z_{i} \otimes\left(p_{1} \cdots p_{i-1}\right) a_{i} b_{i} a_{i}^{-1} y_{i}\right] \\
& +\sum_{i=1}^{n}\left[\sum _ { j = 1 } ^ { i - 1 } \left(\left(p_{1} \cdots p_{j-1}\right)\left(1-a_{j} b_{j} a_{j}^{-1}\right) y_{j} \otimes\left(p_{1} \cdots p_{i-1}\right) a_{i} z_{i}\right.\right. \\
& \left.+\left(p_{1} \cdots p_{j-1}\right) a_{j}\left(1-b_{j} a_{j}^{-1} b_{j}^{-1}\right) z_{j} \otimes\left(p_{1} \cdots p_{i-1}\right) a_{i} z_{i}\right) \\
& \left.+\left(p_{1} \cdots p_{i-1}\right) y_{i} \otimes\left(p_{1} \cdots p_{i-1}\right) a_{i} z_{i}\right]
\end{aligned}
$$




$$
\begin{aligned}
& -\sum_{i=1}^{n-1}\left[\sum_{j=1}^{i}\left(p_{1} \cdots p_{j-1}\right)\left(1-a_{j} b_{j} a_{j}^{-1}\right) y_{j} \otimes\left(p_{1} \cdots p_{i}\right) z_{i}\right. \\
& \left.\quad+\left(p_{1} \cdots p_{j-1}\right) a_{j}\left(1-b_{j} a_{j}^{-1} b_{j}^{-1}\right) z_{j} \otimes\left(p_{1} \cdots p_{i}\right) z_{i}\right] \\
& -z_{n} \otimes b_{n} y_{n} .
\end{aligned}
$$

Proof Let $F$ be the free group generated by $a_{1}, b_{1}, \ldots, a_{n}, b_{n}$, and let $q: F \rightarrow G$ be the canonical projection. We also denote by $q$ the canonical map $\mathbb{Z} F \rightarrow \mathbb{Z} G$. For each element $g \in G$, choose $\widetilde{g} \in F$ such that $q(\widetilde{g})=g$. Given such a choice, we define maps $s_{-1}: \mathbb{Z} \rightarrow P_{0}$ and $s_{0}: P_{0} \rightarrow P_{1}$ by

$$
\begin{aligned}
& s_{-1}(1)=x, \\
& s_{0}(g x)=\sum_{j=1}^{n}\left(q\left(\frac{\partial \widetilde{g}}{\partial a_{j}}\right) y_{j}+q\left(\frac{\partial \widetilde{g}}{\partial b_{j}}\right) z_{j}\right),
\end{aligned}
$$

and by Lemma 1.3 we can find a contracting homotopy for the resolution $P$ such that the maps $s_{-1}$ and $s_{0}$ are as above. The identity $\varepsilon s_{-1}=\mathrm{id}_{\mathbb{Z}}$ trivially holds. The identity $d_{1} s_{0}+s_{-1} d_{0}=\operatorname{id}_{P_{0}}=d_{1} s_{0}(g x)=(g-1) x$ is a consequence of the following identity, which holds for an arbitrary free group $F_{N}=\left\langle c_{1}, c_{2}, \ldots, c_{N}\right\rangle$ :

$$
\sum_{i=1}^{N} \frac{\partial h}{\partial c_{i}}\left(c_{i}-1\right)=h-1 \quad \text { for all } h \in F_{N} .
$$

Let us fix then a choice $g \mapsto \widetilde{g}$ such that

$$
\begin{aligned}
& \widetilde{1}=1 \text {, } \\
& \widetilde{a_{i}}=a_{i} \quad \text { for } 1 \leq i \leq n, \\
& \widetilde{b_{i}}=b_{i} \quad \text { for } 1 \leq i \leq n, \\
& p_{1} \widetilde{\cdots p_{i-1}}=p_{1} \cdots p_{i-1} \quad \text { for } 1 \leq i \leq n, \\
& p_{1} \widetilde{\cdots p_{i-1}} a_{i}=p_{1} \cdots p_{i-1} a_{i} \quad \text { for } 1 \leq i \leq n \text {, } \\
& p_{1} \cdots \bar{p}_{i-1} a_{i} b_{i} a_{i}^{-1}=p_{1} \cdots p_{i-1} a_{i} b_{i} a_{i}^{-1} \quad \text { for } \quad 1 \leq i \leq n-1 \text {. }
\end{aligned}
$$

Once we have $s_{-1}$ and $s_{0}$, we can quickly compute $\Delta_{0}: P_{0} \rightarrow P_{0} \otimes P_{0}$ and $\Delta_{1}: P_{1} \rightarrow$ $(P \otimes P)_{1}$ using Proposition 1.2. We get

$$
\begin{aligned}
\Delta_{0}(x) & =s_{-1} \varepsilon(x) \otimes s_{-1} \varepsilon(x)=x \otimes x \\
\Delta_{1}\left(y_{i}\right) & =\widetilde{s}_{0} \Delta_{0} d_{1}\left(y_{i}\right)=\widetilde{s}_{0}\left(a_{i} x \otimes a_{i} x\right)-\widetilde{s}_{0}(x \otimes x)=y_{i} \otimes a_{i} x+x \otimes y_{i} \\
\Delta_{1}\left(z_{i}\right) & =\widetilde{s}_{0} \Delta_{0} d_{1}\left(z_{i}\right)=\widetilde{s}_{0}\left(b_{i} x \otimes b_{i} x\right)-\widetilde{s}_{0}(x \otimes x)=z_{i} \otimes b_{i} x+x \otimes z_{i} .
\end{aligned}
$$

Now, to calculate the maps $\Delta_{02}: P_{2} \rightarrow P_{0} \otimes P_{2}$ and $\Delta_{11}: P_{2} \rightarrow P_{1} \otimes P_{1}$, we observe the following: if $g, g^{\prime} \in G$, then 


$$
\begin{aligned}
& \widetilde{s}_{1}\left(g x \otimes g^{\prime} y_{i}\right)=\underbrace{s_{0}(g x) \otimes g^{\prime} y_{i}}_{\in P_{1} \otimes P_{1}}+\underbrace{x \otimes s_{1}\left(g^{\prime} y_{i}\right)}_{\in P_{0} \otimes P_{2}}, \\
& \widetilde{s}_{1}\left(g x \otimes g^{\prime} z_{i}\right)=\underbrace{s_{0}(g x) \otimes g^{\prime} z_{i}}_{\in P_{1} \otimes P_{1}}+\underbrace{x \otimes s_{1}\left(g^{\prime} z_{i}\right)}_{\in P_{0} \otimes P_{2}}, \\
& \widetilde{s}_{1}\left(g y_{i} \otimes g^{\prime} x\right)=s_{1}\left(g y_{i}\right) \otimes g^{\prime} x \in P_{2} \otimes P_{0}, \\
& \widetilde{s}_{1}\left(g z_{i} \otimes g^{\prime} x\right)=s_{1}\left(g z_{i}\right) \otimes g^{\prime} x \in P_{2} \otimes P_{0} .
\end{aligned}
$$

For the computation of $\Delta_{11}$, we are interested in the terms that belong to $P_{1} \otimes P_{1}$, and for $\Delta_{02}$ we want the terms belonging to $P_{0} \otimes P_{2}$. So our knowledge of $s_{0}$ is sufficient for the calculation of $\Delta_{11}$. More explicitly, using Proposition 1.2, we have

$$
\begin{aligned}
\Delta_{11}(w)=\pi_{11} \circ \Delta_{2}(w)=\pi_{11} \widetilde{s}_{1}\left(\sum_{i=1}^{n} \frac{\partial p}{\partial a_{i}}\left(x \otimes y_{i}\right)+\frac{\partial p}{\partial b_{i}}\left(x \otimes z_{i}\right)\right) \\
=\pi_{11} \widetilde{s}_{1}\left(\sum_{i=1}^{n}\left(p_{1} \cdots p_{i-1}\right)\left(1-a_{i} b_{i} a_{i}^{-1}\right)\left(x \otimes y_{i}\right)\right. \\
\left.\quad+\left(p_{1} \cdots p_{i-1}\right) a_{i}\left(1-b_{i} a_{i}^{-1} b_{i}^{-1}\right)\left(x \otimes z_{i}\right)\right) \\
=\sum_{i=1}^{n} s_{0}\left(\left(p_{1} \cdots p_{i-1}\right) x\right) \otimes\left(p_{1} \cdots p_{i-1}\right) y_{i} \\
\quad-\sum_{i=1}^{n} s_{0}\left(\left(p_{1} \cdots p_{i-1}\right) a_{i} b_{i} a_{i}^{-1} x\right) \otimes\left(p_{1} \cdots p_{i-1}\right) a_{i} b_{i} a_{i}^{-1} y_{i} \\
\quad+\sum_{i=1}^{n} s_{0}\left(\left(p_{1} \cdots p_{i-1}\right) a_{i} x\right) \otimes\left(p_{1} \cdots p_{i-1}\right) a_{i} z_{i} \\
\quad-\sum_{i=1}^{n} s_{0}\left(\left(p_{1} \cdots p_{i}\right) x\right) \otimes\left(p_{1} \cdots p_{i}\right) z_{i} .
\end{aligned}
$$

Now substituting the expression for $s_{0}$ gives us $\Delta_{11}$. During this computation, we only need to be careful with the term $s_{0}\left(\left(p_{1} \cdots p_{n}\right) a_{n} b_{n} a_{n}^{-1} x\right)=s_{0}\left(b_{n} x\right)=z_{n}$, that had already been defined to calculate $\Delta_{1}$. That is where the term $-z_{n} \otimes b_{n} y_{n}$ in the formula for $\Delta_{11}$ comes from.

Finally, to compute $\Delta_{02}$, we need the map $s_{1}$ of our contracting homotopy $s$. The homomorphism $s_{1}$ must satisfy $s_{1}\left(d_{2}(w)\right)=w$, which is equivalent to

$$
s_{1}\left(\sum_{i=1}^{n} \frac{\partial p}{\partial a_{i}} y_{i}+\frac{\partial p}{\partial b_{i}} z_{i}\right)=w .
$$

But we have 


$$
\begin{aligned}
\Delta_{02}(w)=\pi_{02} \widetilde{s}_{1} \Delta_{1} d_{2}(w) & =\pi_{02} \widetilde{s}_{1}\left(\frac{\partial p}{\partial a_{i}}\left(x \otimes y_{i}\right)+\frac{\partial p}{\partial b_{i}}\left(x \otimes z_{i}\right)\right) \\
& =x \otimes s_{1}\left(\sum_{i=1}^{n} \frac{\partial p}{\partial a_{i}} y_{i}+\frac{\partial p}{\partial b_{i}} z_{i}\right)=x \otimes w .
\end{aligned}
$$

Corollary 2.3 Let $M$ be a trivial $\mathbb{Z} G$-module. If $u, v \in \operatorname{Hom}_{\mathbb{Z} G}\left(P_{1}, M\right)$, then the product $[u] \smile[v] \in H^{2}(G, M \otimes M)$ is represented by the map $(u \smile v) \in$ $\operatorname{Hom}_{\mathbb{Z} G}\left(P_{2}, M \otimes M\right)$ defined by

$$
(u \smile v)(w)=\sum_{i=1}^{n}\left(u\left(y_{i}\right) \otimes v\left(z_{i}\right)-u\left(z_{i}\right) \otimes v\left(y_{i}\right)\right) .
$$

Proof We only need to notice that if $M$ is a trivial $\mathbb{Z} G$-module and $\varepsilon: \mathbb{Z} G \rightarrow \mathbb{Z}$ is the augmentation homomorphism, then for every $f \in \operatorname{Hom}_{\mathbb{Z} G}\left(P_{1}, M\right)$ we have $f(k \alpha)=\varepsilon(k) f(\alpha)$ for all $k \in \mathbb{Z} G, \alpha \in P_{1}$. This, together with the formula we have for $\Delta_{11}$, gives us the desired result.

Now we consider the case of the non-orientable surfaces: let $N_{n}$ be the non-orientable surface with fundamental group given by the presentation

$$
G=\pi_{1}\left(N_{n}\right)=\left\langle a_{1}, \ldots, a_{n} \mid a_{1}^{2} a_{2}^{2} \cdots a_{n}^{2}\right\rangle
$$

for $n \geq 2$. We also define $p=a_{1}^{2} a_{2}^{2} \cdots a_{n}^{2}$. The computations for the case of a nonorientable surface are similar to those we made in the orientable case, and so we simply state the free resolution and the diagonal approximation we get.

Proposition 2.4 (free resolution for the non-orientable case) A free resolution of $\mathbb{Z}$ over $\mathbb{Z} G$ is given by

$$
0 \rightarrow P_{2} \stackrel{d_{2}}{\longrightarrow} P_{1} \stackrel{d_{1}}{\longrightarrow} P_{0} \stackrel{\varepsilon}{\rightarrow} \mathbb{Z} \rightarrow 0
$$

where

$$
P_{0}=\langle x\rangle \cong \mathbb{Z} G, \quad P_{1}=\left\langle y_{1}, \ldots, y_{n}\right\rangle \cong \mathbb{Z} G^{n}, \quad P_{2}=\langle w\rangle \cong \mathbb{Z} G,
$$

and the maps $\varepsilon, d_{1}$ and $d_{2}$ are defined by

$$
\varepsilon(x)=1, \quad d_{1}\left(y_{i}\right)=\left(a_{i}-1\right) x, \quad d_{2}(w)=\sum_{i=1}^{n} \frac{\partial p}{\partial a_{i}} y_{i},
$$

where the partial derivatives are the Fox derivatives.

Theorem 2.5 (diagonal approximation for the non-orientable case) Let $P$ be the free resolution of $\mathbb{Z}$ over $\mathbb{Z} G$ given by Proposition 2.4. A diagonal approximation $\Delta: P \rightarrow$ $(P \otimes P)$ is partially given by 


$$
\begin{gathered}
\Delta_{0}: P_{0} \rightarrow(P \otimes P)_{0} \quad \Delta_{0}(x)=x \otimes x, \\
\Delta_{1}: P_{1} \rightarrow(P \otimes P)_{1} \quad \Delta_{1}\left(y_{i}\right)=y_{i} \otimes a_{i} x+x \otimes y_{i}, \\
\Delta_{02}: P_{2} \rightarrow\left(P_{0} \otimes P_{2}\right) \quad \Delta_{02}(w)=x \otimes w, \\
\Delta_{11}: P_{2} \rightarrow\left(P_{1} \otimes P_{1}\right) \\
\Delta_{11}(w)=\sum_{i=1}^{n}\left[\sum_{j=1}^{i-1}\left(a_{1}^{2} \cdots a_{j-1}^{2}\right)\left(1+a_{j}\right) y_{j} \otimes\left(a_{1}^{2} \cdots a_{i-1}^{2}\right) y_{i}\right. \\
+\sum_{j=1}^{i-1}\left(a_{1}^{2} \cdots a_{j-1}^{2}\right)\left(1+a_{j}\right) y_{j} \otimes\left(a_{1}^{2} \cdots a_{i-1}^{2}\right) a_{i} y_{i} \\
\left.+\left(a_{1}^{2} \cdots a_{i-1}^{2}\right) y_{i} \otimes\left(a_{1}^{2} \cdots a_{i-1}^{2}\right) a_{i} y_{i}\right] .
\end{gathered}
$$

\section{Comments about applications}

Now we make some comments about applications of the results of the previous section. The cohomology ring of a surface group with twisted coefficients has been used in several applications, as stated in the introduction.

\subsection{The cohomology ring of a surface with arbitrary coefficients}

In [9] the cohomology groups of a group which admits a presentation with one single relation was studied. This includes the surface groups for surfaces distinct from $S^{2}$ and $\mathbb{R} P^{2}$. More precisely in [9, Corollary 11.2] Lyndon provides a formula for $H^{n}(G, K)$, $n \geq 2$.

Corollary 3.1 [9] If $G=\left\langle x_{1}, \ldots, x_{m} \mid R\right\rangle$ is defined by the single relation $R$, where $R=Q^{q}$ for no $q>1$, and if $K$ is any left $G$-module, then

$$
H^{2}(G, K)=K /\left(\frac{\partial R}{\partial x_{1}}, \ldots, \frac{\partial R}{\partial x_{m}}\right) K
$$

and $H^{n}(G, K)=0$ for all $n>2$.

Using the resolution of the previous section we can write a formula for $H^{1}$. Let $G$ be a surface group with $G \neq \mathbb{Z}_{2}, G \neq\{1\}$ and let $K$ be a left $G$-module. It is well known that $H^{0}(G, K)=K^{G}$, i.e., the set of elements of $K$ which are $G$-invariant. If $G$ is an orientable surface group, the group cohomology with coefficients $K$, from Sect. 1 , is given by the homology of the complex

$$
0 \rightarrow K \stackrel{d_{1}^{*}}{\longrightarrow} K^{2 n} \stackrel{d_{2}^{*}}{\longrightarrow} K \rightarrow 0,
$$


where $d_{1}^{*}(k)=\left(a_{1}(k)-k, a_{2}(k)-k, \ldots, a_{n}(k)-k, b_{1}(k)-k, \ldots, b_{n}(k)-k\right)$ and $d_{2}^{*}\left(k_{1}, \ldots, k_{2 n}\right)=\sum_{i=1}^{i=n} \partial p\left(k_{i}\right) / \partial a_{i}+\partial p\left(k_{i+n}\right) / \partial b_{i}$. We then get the following proposition.

Proposition 3.2 For $G$ an orientable surface group we have

$$
H^{1}(G, K)=\operatorname{Ker}\left(d_{2}^{*}\right) / d_{1}^{*}\left(K / K^{G}\right),
$$

where $d_{2}^{*}$ is given as above.

Remark 3.3 The calculation of the Fox derivative is straightforward. See, for example, [3].

Remark 3.4 If $G=\mathbb{Z} \oplus \mathbb{Z}$ and $K=\mathbb{Z} \oplus \mathbb{Z}$, let us consider the $\mathbb{Z} G$-module structure given by $\theta(1,0)=\theta(0,1) \in \mathrm{GL}_{2}(\mathbb{Z})$ equal to the matrix

$$
\varphi=\left[\begin{array}{ll}
2 & 1 \\
1 & 1
\end{array}\right] .
$$

Since the matrix has no eigenvalue 1 , it follows that $H^{0}(G, K)=0$. A direct calculation shows that $d_{2}^{*}$ is surjective. So it follows that $H^{2}(G, K)=0$. Also by a straightforward long calculation, we have $H^{1}(G, K)=0$. This phenomena, i.e., the existence of a system of coefficients such that the cohomology is trivial (even the existence of a system of coefficients such that the cohomology is torsion), cannot happen if the surface does not have Euler characteristic zero, at least if the coefficient $K$ is a free abelian group. The reason for that is because the identity map of the chain cellular complex with local coefficients (regarded as abelian groups) will have as a trace the Euler characteristic of the complex times the rank of $K$. This trace is the same as the trace of the homology of the complex which is trivial. So the result follows. For more about cellular homology with local coefficients, see [11].

A similar result holds for the non-orientable surfaces and we leave the details to the reader.

\subsection{Classification of torus bundle over a surface}

Let us consider the $n$-dimensional torus $T^{n}$, which is a Lie group. There is a subfamily of the family of all torus bundles over a given orientable surface $S$ whose elements are the principal torus bundles. It is well known that such bundles are classified by $\left[S, B T^{n}\right]$ (where $B T^{n}$ is the classifying space of the torus), which in turn is $H^{2}(S, \mathbb{Z} \oplus \cdots \oplus \mathbb{Z})=\mathbb{Z} \oplus \cdots \oplus \mathbb{Z}$. In case we consider non-principal bundles, let us focus on those with a prescribed action $\theta: \pi_{1}(S) \rightarrow \operatorname{Aut}(\mathbb{Z} \oplus \cdots \oplus \mathbb{Z})=$ $\mathrm{GL}_{n}(\mathbb{Z})$. Such bundles are classified and they are in one-to-one correspondence with $H^{2}(S, \mathbb{Z} \oplus \cdots \oplus \mathbb{Z})_{\theta}$, the second cohomology group with local coefficients given by $\theta$. See Hilman [7, Section 5, Lemma 5.1] and Robinson [10]. This group is well known and it is isomorphic to the quotient of $\mathbb{Z} \oplus \cdots \oplus \mathbb{Z}$ by the action, i.e., by the subgroup generated by the elements $\theta(g)(x)-x$ for all $g \in \pi_{1}(S)$ and $x \in(\mathbb{Z} \oplus \cdots \oplus \mathbb{Z})$. It 
is easy to find many actions $\theta$ such that the group $H^{2}(S, \mathbb{Z} \oplus \cdots \oplus \mathbb{Z})_{\theta}$ is trivial, i.e., there is only one bundle with that prescribed action.

\subsection{Cohomology of surface bundles over a surface}

The total space $S$ of a surface bundle $S_{1} \rightarrow S \rightarrow S_{2}$ over a surface $S_{2}$ is a 4-manifold. The calculation of the cohomology of those spaces can be approached using spectral sequences. The $E_{2}$ term of the spectral sequence is given by $H^{p}\left(S_{2}, H^{q}\left(S_{1}\right)\right)$ where the cohomology is with local coefficients. The action of $\pi_{1}\left(S_{2}\right)$ on $H^{q}\left(S_{1}\right)$ is completely determined by the action on $H^{1}\left(S_{1}\right)$ and this is a data of the bundle. So one can make use of Sect. 2 to determine the ring structure of $E_{2}$. We point out that this is a relevant step to find the cohomology of the space but in general not a sufficient one.

\subsection{The cohomology ring of a surface with arbitrary coefficients $\mathbb{Z}$}

Here we compute the complete ring structure of the cohomology of a surface for any local system having as group the integers $\mathbb{Z}$. First we consider the case where $G$ is the fundamental group of an orientable surface. If $\widetilde{\mathbb{Z}}$ is a non-trivial $G$-module, then it is proved in [5, Proposition 30] that the only action $\theta: G \rightarrow \operatorname{Aut}(\mathbb{Z})=\{1,-1\}$ we need to consider is the one given by $\theta\left(b_{n}\right)(1)=-1, \theta\left(a_{i}\right)(1)=1$ for all $1 \leq i \leq n$ and $\theta\left(b_{i}\right)(1)=1$ for all $1 \leq i \leq n-1$. We denote by $\widetilde{\mathbb{Z}}$ the $G$-module $\mathbb{Z}$ given by the action $\theta$ and denote by $\mathbb{Z}$ the trivial $G$-module, and we also observe that $\mathbb{Z} \otimes \mathbb{Z} \cong \mathbb{Z}$, $\mathbb{Z} \otimes \widetilde{\mathbb{Z}} \cong \widetilde{\mathbb{Z}}$ and $\widetilde{\mathbb{Z}} \otimes \widetilde{\mathbb{Z}} \cong \mathbb{Z}$. By routine calculation using the diagonal approximation we get

Theorem 3.5 Let $G=\left\langle a_{1}, b_{1}, a_{2}, b_{2}, \ldots, a_{n}, b_{n} \mid\left[a_{1}, b_{1}\right]\left[a_{2}, b_{2}\right] \cdots\left[a_{n}, b_{n}\right]\right\rangle$, for $n \geq 1$. The groups $H^{*}(G, \mathbb{Z})$ and $H^{*}(G, \widetilde{\mathbb{Z}})$ are given by

$$
\begin{array}{ll}
H^{0}(G, \mathbb{Z}) \cong \mathbb{Z}, & H^{0}(G, \widetilde{\mathbb{Z}})=0, \\
H^{1}(G, \mathbb{Z}) \cong \mathbb{Z}^{2 n}, & H^{1}(G, \widetilde{\mathbb{Z}}) \cong \mathbb{Z}^{2 n-2} \oplus \mathbb{Z}_{2}, \\
H^{2}(G, \mathbb{Z}) \cong \mathbb{Z}, & H^{2}(G, \widetilde{\mathbb{Z}}) \cong \mathbb{Z}_{2} .
\end{array}
$$

More precisely, in terms of the free resolution obtained in Proposition 2.1, we have the following sets of generators for the groups $H^{*}(G, \mathbb{Z})$ and $H^{*}(G, \widetilde{\mathbb{Z}})$ :

$$
\begin{aligned}
& \text { generator of } H^{0}(G, \mathbb{Z}):\left\{\left[x^{*}\right]\right\}, \\
& \text { generators of } H^{1}(G, \mathbb{Z}):\left\{\left[y_{1}^{*}\right], \ldots,\left[y_{n}^{*}\right],\left[z_{1}^{*}\right], \ldots,\left[z_{n}^{*}\right]\right\}, \\
& \text { generator of } H^{2}(G, \mathbb{Z}):\left\{\left[w^{*}\right]\right\}, \\
& \text { generators of } H^{1}(G, \widetilde{\mathbb{Z}}):\left\{\left[y_{1}^{*}\right]_{\theta}, \ldots,\left[y_{n-1}^{*}\right]_{\theta},\left[z_{1}^{*}\right]_{\theta}, \ldots,\left[z_{n}^{*}\right]_{\theta}\right\} \\
&\text { (remark: } \left.\left[z_{n}^{*}\right]_{\theta} \text { has order } 2\right), \\
& \text { generator of } H^{2}(G, \widetilde{\mathbb{Z}}):\left\{\left[w^{*}\right]_{\theta}\right\},
\end{aligned}
$$


where $[\cdot]$ and $[\cdot]_{\theta}$ represent cohomology classes in $H^{*}(G, \mathbb{Z})$ and $H^{*}(G, \widetilde{\mathbb{Z}})$, respectively. Finally, we have

$$
\begin{aligned}
& {\left[y_{i}^{*}\right]^{2}=0 \quad \text { for all } 1 \leq i \leq n,} \\
& {\left[z_{i}^{*}\right]^{2}=0 \quad \text { for all } 1 \leq i \leq n,} \\
& {\left[y_{i}^{*}\right] \smile\left[y_{j}^{*}\right]=0 \text {, }} \\
& {\left[z_{i}^{*}\right] \smile\left[z_{j}^{*}\right]=0,} \\
& {\left[y_{i}^{*}\right] \smile\left[z_{j}^{*}\right]= \begin{cases}{\left[w^{*}\right]} & \text { if } i=j \in\{1, \ldots, n\}, \\
0 & \text { if } i \neq j,\end{cases} } \\
& {\left[y_{i}^{*}\right]_{\theta}^{2}=0 \quad \text { for } 1 \leq i \leq n-1 \text {, }} \\
& {\left[z_{i}^{*}\right]_{\theta}^{2}=0 \quad \text { for } 1 \leq i \leq n,} \\
& {\left[y_{i}^{*}\right]_{\theta} \smile\left[z_{i}^{*}\right]_{\theta}=\left[w^{*}\right] \quad \text { for } 1 \leq i \leq n-1 \text {, }} \\
& {\left[y_{i}^{*}\right]_{\theta} \smile\left[z_{j}^{*}\right]_{\theta}=0 \quad \text { if } i \neq j \text {, }} \\
& {\left[y_{i}^{*}\right]_{\theta} \smile\left[y_{j}^{*}\right]_{\theta}=0 \quad \text { if } i \neq j \text {, }} \\
& {\left[z_{i}^{*}\right]_{\theta} \smile\left[z_{j}^{*}\right]_{\theta}=0 \quad \text { if } i \neq j \text {, }} \\
& {\left[y_{i}^{*}\right] \smile\left[y_{j}^{*}\right]_{\theta}=0 \quad \text { for all }(i, j) \in\{1, \ldots, n\} \times\{1, \ldots, n-1\} \text {, }} \\
& {\left[z_{i}^{*}\right] \smile\left[z_{j}^{*}\right]_{\theta}=0 \quad \text { for all } i, j \in\{1, \ldots, n\} \text {, }} \\
& {\left[y_{i}^{*}\right] \smile\left[z_{j}^{*}\right]_{\theta}= \begin{cases}{\left[w^{*}\right]_{\theta}} & \text { if } i=j \in\{1, \ldots, n\}, \\
0 & \text { otherwise. }\end{cases} }
\end{aligned}
$$

The non-orientable case is similar. Let $G=\left\langle a_{1}, \ldots, a_{n} \mid a_{1}^{2} a_{2}^{2} \cdots a_{n}^{2}\right\rangle$ for $n \geq 2$. Using similar arguments as in [5, Proposition 32], we can get that the number of equivalent classes of structures of $\mathbb{Z}$ as a $G$-module is 4 if $n>2$, and is 3 if $n=2$. More precisely:

a) for the first class choose as representative the trivial action $\theta_{0}: G \rightarrow \operatorname{Aut}(\mathbb{Z})$;

b) for the second class choose as representative the action $\theta_{1}: G \rightarrow \operatorname{Aut}(\mathbb{Z})$, defined by $\theta_{1}\left(a_{1}\right)(1)=-1$ and $\theta_{1}\left(a_{i}\right)(1)=1$ for $2 \leq i \leq n$;

c) for the third class choose as representative the action $\theta_{2}: G \rightarrow \operatorname{Aut}(\mathbb{Z})$, defined in case $n>2$ by $\theta_{2}\left(a_{1}\right)(1)=\theta_{2}\left(a_{2}\right)(1)=-1$ and $\theta_{2}\left(a_{i}\right)(1)=1$ for $3 \leq i \leq n$;

d) for the fourth class choose as representative the action $\theta_{3}: G \rightarrow \operatorname{Aut}(\mathbb{Z})$, defined by $\theta_{3}\left(a_{i}\right)(1)=-1$ for $1 \leq i \leq n$.

To see why any structure is equivalent to one of the four above $\theta$, we follow [1]. Of course it suffices to consider $n>3$. Let $\varphi: G \rightarrow \operatorname{Aut}(\mathbb{Z})=\{1,-1\}$ where $\varphi\left(a_{i}\right) \neq 1$ for some $i$. The main step is to consider the change of variables $d^{\prime}=b c d, a^{\prime}=$ $a^{2} b^{2} c b^{-1} a^{-1}, c^{\prime}=a b d^{-1}, b^{\prime}=a^{\prime-1} a c^{\prime-1}$, which satisfies $a^{\prime 2} b^{\prime 2} c^{\prime 2} d^{\prime 2}=a^{2} b^{2} c^{2} d^{2}$ and $(\varphi(a), \varphi(b), \varphi(c), \varphi(d))=(1,-1,-1,-1) \Rightarrow\left(\varphi\left(a^{\prime}\right), \varphi\left(b^{\prime}\right), \varphi\left(c^{\prime}\right), \varphi\left(d^{\prime}\right)\right)=$ $(1,1,1,-1)$. So we can reduce any given structure to one of the four structures given above. In particular, 


$$
\mathbb{Z}_{\theta_{1}} \otimes \mathbb{Z}_{\theta_{3}} \cong\left\{\begin{array} { l l } 
{ \mathbb { Z } _ { \theta _ { 1 } } } & { \text { if } n \text { is even, } } \\
{ \mathbb { Z } _ { \theta _ { 2 } } } & { \text { if } n \text { is odd, } }
\end{array} \quad \mathbb { Z } _ { \theta _ { 2 } } \otimes \mathbb { Z } _ { \theta _ { 3 } } \cong \left\{\begin{array}{ll}
\mathbb{Z}_{\theta_{2}} \text { if } n \text { is even }, \\
\mathbb{Z}_{\theta_{1}} \text { if } n \text { is odd. }
\end{array}\right.\right.
$$

With a little more of work, it is not hard to show that the four structures above are not equivalent. By routine calculation using the diagonal approximation we get

Theorem 3.6 Let $G=\left\langle a_{1}, \ldots, a_{n} \mid a_{1}^{2} a_{2}^{2} \cdots a_{n}^{2}\right\rangle$, where $n \geq 2$. The cohomology groups $H^{*}\left(G, \mathbb{Z}_{\theta_{i}}\right)$ are given by

$$
\begin{array}{lll}
H^{0}\left(G, \mathbb{Z}_{\theta_{0}}\right) \cong \mathbb{Z}, & H^{1}\left(G, \mathbb{Z}_{\theta_{0}}\right) \cong \mathbb{Z}^{n-1}, & H^{2}\left(G, \mathbb{Z}_{\theta_{0}}\right) \cong \mathbb{Z}_{2}, \\
H^{0}\left(G, \mathbb{Z}_{\theta_{1}}\right)=0, & H^{1}\left(G, \mathbb{Z}_{\theta_{1}}\right) \cong \mathbb{Z}^{n-2} \oplus \mathbb{Z}_{2}, & H^{2}\left(G, \mathbb{Z}_{\theta_{1}}\right) \cong \mathbb{Z}_{2}, \\
H^{0}\left(G, \mathbb{Z}_{\theta_{2}}\right)=0, & H^{1}\left(G, \mathbb{Z}_{\theta_{2}}\right) \cong \mathbb{Z}^{n-2} \oplus \mathbb{Z}_{2}, & H^{2}\left(G, \mathbb{Z}_{\theta_{2}}\right) \cong \mathbb{Z}_{2}, \\
H^{0}\left(G, \mathbb{Z}_{\theta_{3}}\right)=0, & H^{0}\left(G, \mathbb{Z}_{\theta_{3}}\right) \cong \mathbb{Z}^{n-1} \oplus \mathbb{Z}_{2}, & H^{0}\left(G, \mathbb{Z}_{\theta_{3}}\right) \cong \mathbb{Z} .
\end{array}
$$

More precisely, in terms of the free resolution obtained in Proposition 2.4, we can determine explicit generators for the groups $H^{*}\left(G, \mathbb{Z}_{\theta_{i}}\right)$ as follows:

$$
\begin{aligned}
& H^{0}\left(G, \mathbb{Z}_{\theta_{0}}\right)=\left\langle\left[x^{*}\right]_{\theta_{0}}\right\rangle, \\
& H^{1}\left(G, \mathbb{Z}_{\theta_{0}}\right)=\bigoplus_{k=1}^{n-1}\left\langle\left[y_{k}^{*}-y_{k+1}^{*}\right]_{\theta_{0}}\right\rangle, \\
& H^{2}\left(G, \mathbb{Z}_{\theta_{0}}\right)=\left\langle\left[w^{*}\right]_{\theta_{0}}\right\rangle, \\
& H^{0}\left(G, \mathbb{Z}_{\theta_{1}}\right)=0, \\
& H^{1}\left(G, \mathbb{Z}_{\theta_{1}}\right)=\left\langle\left[y_{1}^{*}\right]_{\theta_{1}}\right\rangle \oplus \bigoplus_{k=2}^{n-1}\left\langle\left[y_{k}^{*}-y_{k+1}^{*}\right]_{\theta_{1}}\right\rangle, \\
& H^{2}\left(G, \mathbb{Z}_{\theta_{1}}\right)=\left\langle\left[w^{*}\right]_{\theta_{1}}\right\rangle, \\
& H^{0}\left(G, \mathbb{Z}_{\theta_{2}}\right)=0, \\
& H^{1}\left(G, \mathbb{Z}_{\theta_{2}}\right)=\left\langle\left[y_{1}^{*}\right]_{\theta_{2}}\right\rangle \oplus\left\langle\left[y_{1}^{*}+y_{2}^{*}\right]_{\theta_{2}}\right\rangle \oplus \bigoplus_{k=3}^{n-1}\left\langle\left[y_{k}^{*}-y_{k+1}^{*}\right]_{\theta_{2}}\right\rangle, \\
& H^{2}\left(G, \mathbb{Z}_{\theta_{2}}\right)=\left\langle\left[w^{*}\right]_{\theta_{2}}\right\rangle, \\
& H^{0}\left(G, \mathbb{Z}_{\theta_{3}}\right)=0, \\
& H^{1}\left(G, \mathbb{Z}_{\theta_{3}}\right)=\left\langle\left[y_{1}^{*}+\cdots+y_{n}^{*}\right]_{\theta_{3}}\right\rangle \oplus \bigoplus_{k=1}^{n-1}\left\langle\left[y_{k}^{*}\right]_{\theta_{3}}\right\rangle, \\
& H^{2}\left(G, \mathbb{Z}_{\theta_{3}}\right)=\left\langle\left[w^{*}\right]_{\theta_{3}}\right\rangle,
\end{aligned}
$$

where $[\cdot]_{\theta_{i}}$ represents the cohomology class in $H^{*}\left(G, \mathbb{Z}_{\theta_{i}}\right)$ and the elements $\left[y_{1}^{*}\right]_{\theta_{1}},\left[y_{1}^{*}+y_{2}^{*}\right]_{\theta_{2}},\left[y_{1}^{*}+\cdots+y_{n}^{*}\right]_{\theta_{3}},\left[w^{*}\right]_{\theta_{1}}$ and $\left[w^{*}\right]_{\theta_{2}}$ have order 2 . Finally, the products

$$
H^{1}\left(G, \mathbb{Z}_{\theta_{i}}\right) \otimes H^{1}\left(G, \mathbb{Z}_{\theta_{j}}\right) \stackrel{\smile}{\longrightarrow} H^{2}\left(G, \mathbb{Z}_{\theta_{i}} \otimes \mathbb{Z}_{\theta_{j}}\right)
$$


are given by

$$
\begin{aligned}
& {\left[y_{k}^{*}-y_{k+1}^{*}\right]_{\theta_{0}}^{2}=0 \text {, }} \\
& {\left[y_{k}^{*}-y_{k+1}^{*}\right]_{\theta_{0}} \smile\left[y_{\ell}^{*}-y_{\ell+1}^{*}\right]_{\theta_{0}}= \begin{cases}{\left[w^{*}\right]_{\theta_{0}}} & \text { if } \ell=k \pm 1, \\
0 & \text { otherwise, }\end{cases} } \\
& {\left[y_{k}^{*}-y_{k+1}^{*}\right]_{\theta_{0}} \smile\left[y_{1}^{*}\right]_{\theta_{1}}= \begin{cases}{\left[w^{*}\right]_{\theta_{1}}} & \text { if } k=1, \\
0 & \text { if } k>1,\end{cases} } \\
& {\left[y_{k}^{*}-y_{k+1}^{*}\right]_{\theta_{0}} \smile\left[y_{\ell}^{*}-y_{\ell+1}^{*}\right]_{\theta_{1}}= \begin{cases}{\left[w^{*}\right]_{\theta_{1}}} & \text { if } \ell=k \pm 1, \\
0 & \text { otherwise, }\end{cases} } \\
& {\left[y_{k}^{*}-y_{k+1}^{*}\right]_{\theta_{0}} \smile\left[y_{\ell}^{*}-y_{\ell+1}^{*}\right]_{\theta_{2}}= \begin{cases}{\left[w^{*}\right]_{\theta_{2}}} & \text { if } \ell=k \pm 1, \\
0 & \text { otherwise, }\end{cases} } \\
& {\left[y_{k}^{*}-y_{k+1}^{*}\right]_{\theta_{0}} \smile\left[y_{1}^{*}\right]_{\theta_{2}}= \begin{cases}{\left[w^{*}\right]_{\theta_{2}}} & \text { if } k=1, \\
0 & \text { otherwise }\end{cases} } \\
& {\left[y_{k}^{*}-y_{k+1}^{*}\right]_{\theta_{0}} \smile\left[y_{1}^{*}+y_{2}^{*}\right]_{\theta_{2}}= \begin{cases}{\left[w^{*}\right]_{\theta_{2}}} & \text { if } k=2, \\
0 & \text { otherwise, }\end{cases} } \\
& {\left[y_{1}^{*}\right]_{\theta_{1}}^{2}=\left[w^{*}\right]_{\theta_{0}},} \\
& {\left[y_{k}^{*}-y_{k+1}^{*}\right]_{\theta_{1}}^{2}=0 \text {, }} \\
& {\left[y_{1}^{*}\right]_{\theta_{1}} \smile\left[y_{k}^{*}-y_{k+1}^{*}\right]_{\theta_{1}}=0,} \\
& {\left[y_{1}^{*}\right]_{\theta_{2}}^{2}=\left[w^{*}\right]_{\theta_{0}} \text {, }} \\
& {\left[y_{1}^{*}+y_{2}^{*}\right]_{\theta_{2}}^{2}=0 \text {, }} \\
& {\left[y_{1}^{*}\right]_{\theta_{2}} \smile\left[y_{1}^{*}+y_{2}^{*}\right]_{\theta_{2}}=\left[w^{*}\right]_{\theta_{0}},} \\
& {\left[y_{1}^{*}\right]_{\theta_{2}} \smile\left[y_{k}^{*}-y_{k+1}^{*}\right]_{\theta_{2}}=0 \text {, }} \\
& {\left[y_{1}^{*}+y_{2}^{*}\right]_{\theta_{2}} \smile\left[y_{k}^{*}-y_{k+1}^{*}\right]_{\theta_{2}}=0 \text {, }} \\
& {\left[y_{1}^{*}\right]_{\theta_{1}} \smile\left[y_{1}^{*}\right]_{\theta_{2}}=\left[w^{*}\right]_{\theta_{1}} \text {, }} \\
& {\left[y_{1}^{*}\right]_{\theta_{1}} \smile\left[y_{1}^{*}+y_{2}^{*}\right]_{\theta_{2}}=\left[w^{*}\right]_{\theta_{1}} \text {, }} \\
& {\left[y_{1}^{*}\right]_{\theta_{1}} \smile\left[y_{\ell}^{*}-y_{\ell+1}^{*}\right]_{\theta_{2}}=0 \text {, }} \\
& {\left[y_{k}^{*}-y_{k+1}^{*}\right]_{\theta_{1}} \smile\left[y_{1}^{*}\right]_{\theta_{2}}=0 \text {, }} \\
& {\left[y_{k}^{*}-y_{k+1}^{*}\right]_{\theta_{1}} \smile\left[y_{1}^{*}+y_{2}^{*}\right]_{\theta_{2}}= \begin{cases}{\left[w^{*}\right]_{\theta_{1}}} & \text { if } k=2, \\
0 & \text { otherwise, }\end{cases} } \\
& {\left[y_{k}^{*}-y_{k+1}^{*}\right]_{\theta_{1}} \smile\left[y_{\ell}^{*}+y_{\ell+1}^{*}\right]_{\theta_{2}}= \begin{cases}{\left[w^{*}\right]_{\theta_{1}}} & \text { if } \ell=k \pm 1, \\
0 & \text { otherwise, }\end{cases} } \\
& {\left[y_{k}^{*}\right]_{\theta_{3}}^{2}=\left[w^{*}\right]_{\theta_{0}},} \\
& {\left[y_{1}^{*}+\cdots+y_{n}^{*}\right]_{\theta_{3}}^{2}=n\left[w^{*}\right]_{\theta_{0}} \text {, }} \\
& {\left[y_{1}^{*}+\cdots+y_{n}^{*}\right]_{\theta_{3}} \smile\left[y_{k}^{*}\right]_{\theta_{3}}=\left[w^{*}\right]_{\theta_{0}} \text {, }}
\end{aligned}
$$




$$
\begin{aligned}
& {\left[y_{k}^{*}-y_{k+1}^{*}\right]_{\theta_{0}} \smile\left[y_{\ell}^{*}\right]_{\theta_{3}}= \begin{cases}{\left[w^{*}\right]_{\theta_{3}}} & \text { if } \ell \in\{k, k+1\}, \\
0 & \text { otherwise, }\end{cases} } \\
& {\left[y_{k}^{*}-y_{k+1}^{*}\right]_{\theta_{0}} \smile\left[y_{1}^{*}+\cdots+y_{n}^{*}\right]_{\theta_{3}}=0 \text {, }} \\
& {\left[y_{1}^{*}\right]_{\theta_{1}} \smile\left[y_{k}^{*}\right]_{\theta_{3}}= \begin{cases}{\left[w^{*}\right]_{\zeta}} & \text { if } k=1, \\
0 & \text { if } k \neq 1,\end{cases} } \\
& {\left[y_{1}^{*}\right]_{\theta_{1}} \smile\left[y_{1}^{*}+\cdots+y_{n}^{*}\right]_{\theta_{3}}=\left[w^{*}\right]_{\zeta} \text {, }} \\
& {\left[y_{k}^{*}-y_{k+1}^{*}\right]_{\theta_{1}} \smile\left[y_{\ell}^{*}\right]_{\theta_{3}}= \begin{cases}{\left[w^{*}\right]_{\zeta}} & \text { if } \ell \in\{k, k+1\}, \\
0 & \text { otherwise, }\end{cases} } \\
& {\left[y_{k}^{*}-y_{k+1}^{*}\right]_{\theta_{1}} \smile\left[y_{1}^{*}+\cdots+y_{n}^{*}\right]_{\theta_{3}}=0 \text {, }} \\
& {\left[y_{1}^{*}\right]_{\theta_{2}} \smile\left[y_{k}^{*}\right]_{\theta_{3}}= \begin{cases}{\left[w^{*}\right]_{\xi}} & \text { if } k=1, \\
0 & \text { if } k \neq 1,\end{cases} } \\
& {\left[y_{1}^{*}\right]_{\theta_{2}} \smile\left[y_{1}^{*}+\cdots+y_{n}^{*}\right]_{\theta_{3}}=\left[w^{*}\right]_{\xi} \text {, }} \\
& {\left[y_{1}^{*}+y_{2}^{*}\right]_{\theta_{2}} \smile\left[y_{k}^{*}\right]_{\theta_{3}}= \begin{cases}{\left[w^{*}\right]_{\xi}} & \text { if } k \in\{1,2\}, \\
0 & \text { otherwise, }\end{cases} } \\
& {\left[y_{1}^{*}+y_{2}^{*}\right]_{\theta_{2}} \smile\left[y_{1}^{*}+\cdots+y_{n}^{*}\right]_{\theta_{3}}=0 \text {, }} \\
& {\left[y_{k}^{*}-y_{k+1}^{*}\right]_{\theta_{2}} \smile\left[y_{\ell}^{*}\right]_{\theta_{3}}= \begin{cases}{\left[w^{*}\right]_{\xi}} & \text { if } \ell \in\{k, k+1\}, \\
0 & \text { otherwise, }\end{cases} } \\
& {\left[y_{k}^{*}-y_{k+1}^{*}\right]_{\theta_{2}} \smile\left[y_{1}^{*}+\cdots+y_{n}^{*}\right]_{\theta_{3}}=0 \text {, }}
\end{aligned}
$$

where $\zeta$ is $\theta_{1}$ if $n$ is even and $\theta_{2}$ if $n$ is odd and $\xi$ is $\theta_{2}$ if $n$ is even and $\theta_{1}$ if $n$ is odd.

Remark 3.7 The cohomology ring structure with arbitrary coefficients of the groups of the form $G \rtimes \mathbb{Z}_{2}$, where $G$ is a surface group, seems an interesting problem. By [8, Proposition 7.1] those groups are potential candidates to act freely and properly on some even dimensional homotopy spheres. Since these groups are infinite, the homotopy sphere where the groups may act cannot be compact, which is for example the case of $R^{m} \times S^{2 n}$. Hence certain cohomological properties of those groups are expected to show up. We hope to pursue this idea somewhere.

Acknowledgments We would like to thank the referee for his/her comments which helped to improve the presentation of this work.

\section{References}

1. Bauval, A.: Reidemeister-Schreier method. Private communication

2. Brown, K.S.: Cohomology of Groups. Graduate Texts in Mathematics, vol. 87. Springer, New York (1982)

3. Fadell, E., Husseini, S.: The Nielsen number on surfaces. In: Topological Methods in Nonlinear Functional Analysis (Toronto, 1982). Contemp. Math., vol. 21, pp. 59-98. American Mathematical Society, Providence (1983) 
4. Gonçalves, D.L., de Oliveira, E.: The Lefschetz coincidence number for maps among compact surfaces. Far East J. Math. Sci., special volume, part II, 147-166 (1997)

5. Gonçalves, D.L., Guaschi, J.: The Borsuk-Ulam theorem for maps into a surface. Topol. Appl. 157(1011), 1742-1759 (2010)

6. Handel, D.: On products in the cohomology of the dihedral groups. Tohoku Math. J. 45(1), 13-42 (1993)

7. Hillman, J.A.: Four-manifolds, Geometries and Knots. Geometry \& Topology Monographs, vol. 5. Geometry \& Topology Publications, Coventry (2002)

8. Lee, J.B.: Transformation groups on $S^{n} \times \mathbb{R}^{m}$. Topol. Appl. 53(2), 187-204 (1993)

9. Lyndon, R.C.: Cohomology theory of groups with a single defining relation. Ann. Math. 52(3), 650-665 (1950)

10. Robinson, C.A.: Moore-Postnikov systems for non-simple fibrations. Ill. J. Math. 16(2), 234-242 (1972)

11. Whitehead, G.W.: Elements of Homotopy Theory. Graduate Texts in Mathematics, vol. 61. Springer, New York (1978) 